\title{
Adjuvant chemotherapy for luminal A breast cancer: a prospective study comparing two popular chemotherapy regimens
}

This article was published in the following Dove Press journal:

OncoTargets and Therapy

8 August 2013

Number of times this article has been viewed

\author{
Yasser Abdel Kader \\ Tamer El-Nahas \\ Amr Sakr \\ Department of Clinical Oncology, \\ Cairo University, Cairo, Egypt
}

\begin{abstract}
Introduction: Based on the variable benefit of taxanes in the adjuvant setting of early breast cancer in certain tumor phenotypes, especially in human epidermal growth factor receptor (HER)2-positive and triple-negative disease, and with the observation of a lesser benefit in luminal $\mathrm{A}$, this research article aimed at exploring the value of docetaxel in patients with an estrogen receptor-positive, HER2-negative disease phenotype, who might not derive the same benefits as those with other phenotypes.
\end{abstract}

Patients and methods: This was a randomized prospective study comparing disease-free survival (DFS) and safety profile of sequential adjuvant three cycles Fluorouracil, Epirubicin, Cyclophosphamide followed by three cycles Docetaxel (FEC-D) versus six cycles classic Fluorouracil, Epirubicin, Cyclophosphamide (FEC)-100 in 60 Egyptian women who presented to Dar Al Fouad Hospital during the period June 2007 to July 2008 with (pT1-2 pN0-3 M0). The primary end point was DFS in a follow-up period of 4 years. The secondary end point was toxicity profile.

Results: Four-year DFS rates were comparable in both arms: $73.3 \% \pm 8.1 \%$ in the FEC-D arm versus $76.5 \% \pm 7.8 \%$ in the FEC-100 arm $(P=0.83)$. N3 and grade III subgroups achieved the worst DFS in both subgroups ( $P=0.001$ and $P=0.214$, respectively). The rate of nausea and vomiting was higher in the FEC-100 arm $(P=0.49)$, while grade III-IV neutropenia and febrile neutropenia incidence was similar between both arms.

Conclusion: Sequential adjuvant chemotherapy with FEC followed by docetaxel achieved comparable DFS results to FEC alone in luminal A phenotype subgroups of breast cancer.

Keywords: taxane, epirubicin, adjuvant, breast cancer

\section{Introduction}

Over the last 30 years, adjuvant chemotherapy has improved survival for women with early breast cancer. Since the 1990s, anthracycline-based chemotherapy has proved to be superior to classic cyclophosphamide, methotrexate, and fluorouracil (CMF). ${ }^{1,2}$ Incorporation of a taxane (paclitaxel or docetaxel) offered further improvement to patient outcomes in the adjuvant setting. ${ }^{3,4}$

Clinical trials incorporating taxanes in the adjuvant setting of breast cancer have not shown the same clinical outcome. ${ }^{3,4}$ In some studies, evaluation of taxane benefit was difficult to make because of different population sizes, inclusion of all tumor phenotypes, and biologically heterogeneous populations, and since different taxane schedules were compared with different anthracycline control regimens of often unequal duration. ${ }^{5}$ In another trial, many patient subgroups gained less benefit from additional taxanes, especially in regard to overall survival (OS). ${ }^{6}$ Another significant role for adjuvant taxanes sequential to anthracyclines in the adjuvant setting is reducing long-term adverse events (such as induction of leukemia and cardiotoxicity) to
Correspondence: Tamer El-Nahas Department of Clinical Oncology, Cairo University, I Al Saray Street, Al Manial, Cairo, Egypt Fax +20233477771

Email elnahas@sphinxcure.com 
a minimum by reducing exposure to cumulative doses of anthracyclines.

The value of adjuvant taxane to breast cancer outcomes in luminal A patients is affected by events in the early years and further follow-up is required before a possible longerterm benefit might be witnessed or excluded in this patient population, since the risk of relapse continues for at least 15 years after diagnosis for these patients, which is partly attributed to consequences of effective endocrine therapy for patients with estrogen receptor (ER)-positive disease.

ER as a biological marker seems to have little predictive value in determining taxane responsiveness. ${ }^{7-9}$ The CALGB 9344 trial showed that the benefits of paclitaxel occurred mainly in patients with ER-negative or human epidermal growth factor receptor (HER)2-positive tumors, with less gain in the bigger subgroup, (ER positive or Her2 negative groups) of patients, especially those with the ER-positive/ HER2-negative phenotype. ${ }^{10}$ In a similar trial, docetaxel benefit was most evident in patients whose tumors were ERnegative and HER2-positive, with other subgroups deriving less or no apparent benefit. ${ }^{10}$ Therefore, it is postulated that luminal A patients might not necessarily gain a clinically worthwhile benefit from taxanes. Finally, in view of the molecular diversity of breast cancer, adjuvant taxanes have provided different benefits for special patient subgroups, especially those who are HER2-positive and triple negative. ${ }^{10}$ Therefore, there is a need to identify patients who would not necessarily obtain benefit from this treatment.

This study was conducted with the aim of evaluating the value of adjuvant sequential taxanes, compared to six cycles of classic anthracyclines, in patients with the luminal A phenotype in a small-sized Egyptian population.

\section{Patients and methods}

This was a randomized prospective study comparing diseasefree survival (DFS) and toxicity of adjuvant taxanes in 60 females (aged $>18$ years) who presented at Dar Al Fouad Hospital, Egypt, during the period from June 2007 to July 2008 with stage (pT 1-2 pN 0-3 M0). Inclusion criteria were normal hematological, hepatic, and renal function. Patients were excluded if they had a history of cardiac disease, locally advanced or metastatic disease, bilateral breast cancer, pregnancy, or a previous history of cancer.

Patients were randomly allocated with closed envelope into the treatment groups at a ratio of 1:1 using a computer system to three cycles of Fluorouracil, Epirubicin, Cyclophosphamide (FEC)-100 followed by three cycles docetaxel (Arm I) or six cycles of FEC-100 (Arm II). Thirty patients were assigned to each arm.

\section{Treatments}

Patients in Arm I received three cycles of FEC, comprising cyclophosphamide $500 \mathrm{mg} / \mathrm{m}^{2}$, epirubicin $100 \mathrm{mg} / \mathrm{m}^{2}$, and 5 -fluorouracil $500 \mathrm{mg} / \mathrm{m}^{2}$ and three cycles of Taxotere ${ }^{\circledR}$ (Sanofi-Aventis, Bridgewater, NJ, USA), comprising docetaxel $100 \mathrm{mg} / \mathrm{m}^{2}$.

In Arm II, patients received six cycles of FEC, comprising cyclophosphamide $500 \mathrm{mg} / \mathrm{m}^{2}$, epirubicin $100 \mathrm{mg} / \mathrm{m}^{2}$, and 5 -fluorouracil $500 \mathrm{mg} / \mathrm{m}^{2}$.

\section{Adjuvant treatment after chemotherapy completion}

Adjuvant tamoxifen $20 \mathrm{mg} /$ day to pre- or postmenopausal women or aromatase inhibitor to postmenopausal women was started after chemotherapy for patients not subjected to radiotherapy otherwise following radiotherapy and continued for 5 years.

Radiotherapy was initiated within 4 weeks after the last cycle of chemotherapy and was mandatory for all patients who had undergone breast-conserving surgery. Radiation to the chest wall, supraclavicular area, and internal mammary chain was recommended following mastectomy to indicated patients. Irradiation of the axilla was prohibited. Radiotherapy procedures were similar for both arms.

\section{Evaluations}

The tolerability of chemotherapy was evaluated before each cycle. In addition, an absolute blood count was performed on day 21, and nonhematologic toxicity was evaluated during the period between cycles. Toxicity was graded according to National Cancer Institute Common Toxicity Criteria for Adverse Events version 3.0. ${ }^{11}$ The resting left ventricle ejection fraction (LVEF) was measured by echocardiographic methods at baseline. A physical examination was performed every 4 months for the first 2 years, then every 6 months for the following 2 years. Imaging studies (ie, mammography, chest X-ray, liver ultrasound, and bone scan) were performed 1 year after the initial surgery, then yearly thereafter for 5 years. Beyond this period, a mammography was performed annually.

The primary end point of the study was 4 years of DFS, which was defined as the time from randomization until first relapse (local, regional, or distant), contralateral breast cancer, or death from any cause. The secondary end point was toxicity profile.

\section{Statistical analysis}

Comparison of toxicity was done using Fisher's exact test and survival was estimated using Kaplan-Meier and log rank for comparing curves. $P$-values were always two-tailed and significance was at the 0.05 level. 


\section{Results}

Between June 2007 and July 2008, 60 women were enrolled at Dar Al Fouad Hospital (30 in the FEC-D arm and 30 in the FEC arm). Baseline patient information and disease characteristics are shown in Table 1.

\section{Efficacy results}

Four-year DFS rates (Figure 1) were similar in both arms, at $73.3 \% \pm 8.1 \%$ in the FEC-D arm versus $76.5 \% \pm 7.8 \%$ in the FEC-100 arm $(P=0.83)$.

In both treatment arms, postmenopausal women (Figure 2) achieved slightly better 4-year DFS (78.8\% $\pm 7.1 \%)$ compared to premenopausal women $(70 \% \pm 8.8 \%)(P=0.364)$. The 4-year DFS was almost identical in T1 patients $(71.4 \% \pm 12 \%)$ and T2 patients $(75.8 \% \pm 6.3 \%)(P=0.89)$.

N3 patients (Figure 3) achieved the worst 4-year DFS, at $25 \% \pm 1.5 \%$, compared to those at N0-N2 $(P=0.001)$ in both treatment arms. Grade III patients (Figure 4) achieved inferior survival rates, at $58.3 \% \pm 14.2 \%$, compared to grade II patients $(79 \% \pm 5.8 \%)(P=0.214)$.

Four-year DFS was almost identical in the group receiving antihormonal treatment as tamoxifen $(77 \% \pm 7.1 \%)$ as in the aromatase inhibition group $(72 \% \pm 8.9 \%)(P=0.7)$.

Table I Baseline patient and disease characteristics of randomly assigned patients

\begin{tabular}{|c|c|c|c|}
\hline & $\begin{array}{l}\text { FEC-D } \\
\text { group }(n=30)\end{array}$ & $\begin{array}{l}\text { FEC } \\
\text { group }(n=30)\end{array}$ & \\
\hline \multicolumn{4}{|l|}{ Age, years } \\
\hline Range & $28-69$ & $29-68$ & $P=0.93$ \\
\hline Mean & 49.97 & 49.7 & \\
\hline \multicolumn{4}{|l|}{ Menopause, n (\%) } \\
\hline Pre & I3/30 (43.3) & $14 / 30(46.7)$ & $P=0.79$ \\
\hline Post & $17 / 30(56.7)$ & $16 / 30(53.3 \%)$ & \\
\hline \multicolumn{4}{|l|}{ Surgery, n (\%) } \\
\hline Conservative & $16 / 30(53.3)$ & $16 / 30(53.3)$ & $P=1.0$ \\
\hline Radical & I4/30 (46.7) & I4/30 (64.7) & \\
\hline \multicolumn{4}{|l|}{ T-stage, n (\%) } \\
\hline TI & $7 / 30(23.3)$ & $7 / 30(23.3)$ & $P=1.0$ \\
\hline $\mathrm{T} 2$ & $23 / 30(76.7)$ & $23 / 30(76.6)$ & \\
\hline \multicolumn{4}{|l|}{ N-stage, n (\%) } \\
\hline No & $3 / 30(10)$ & $4 / 30(13.3)$ & $P=0.9$ \\
\hline $\mathrm{NI}$ & I3/30 (43.4) & $12 / 30(40)$ & \\
\hline N2-3 & $14 / 30(46.6)$ & $14 / 30(46.6)$ & \\
\hline \multicolumn{4}{|l|}{ Grade, n (\%) } \\
\hline ॥ & $24 / 30(80)$ & $25 / 30(83.3)$ & $P=0.74$ \\
\hline III & $6 / 30(20)$ & $5 / 30(16.7)$ & \\
\hline \multicolumn{4}{|c|}{ Radiotherapy, n (\%) } \\
\hline Yes & $22 / 30(73.3)$ & $21 / 30(70)$ & $P=0.77$ \\
\hline No & $8 / 30(26.7)$ & $9 / 30(30)$ & \\
\hline \multicolumn{4}{|c|}{ Adjuvant hormone, $\mathrm{n}(\%)$} \\
\hline Tamoxifen & $17 / 30(56.7)$ & $18 / 30(60)$ & $P=0.79$ \\
\hline Aromatase & 13/30 (43.3) & $12 / 30(30)$ & \\
\hline
\end{tabular}

Abbreviations: FEC, Fluorouracil, Epirubicin, Cyclophosphamide; FEC-D, Fluorouracil, Epirubicin, Cyclophosphamide - Docetaxel.

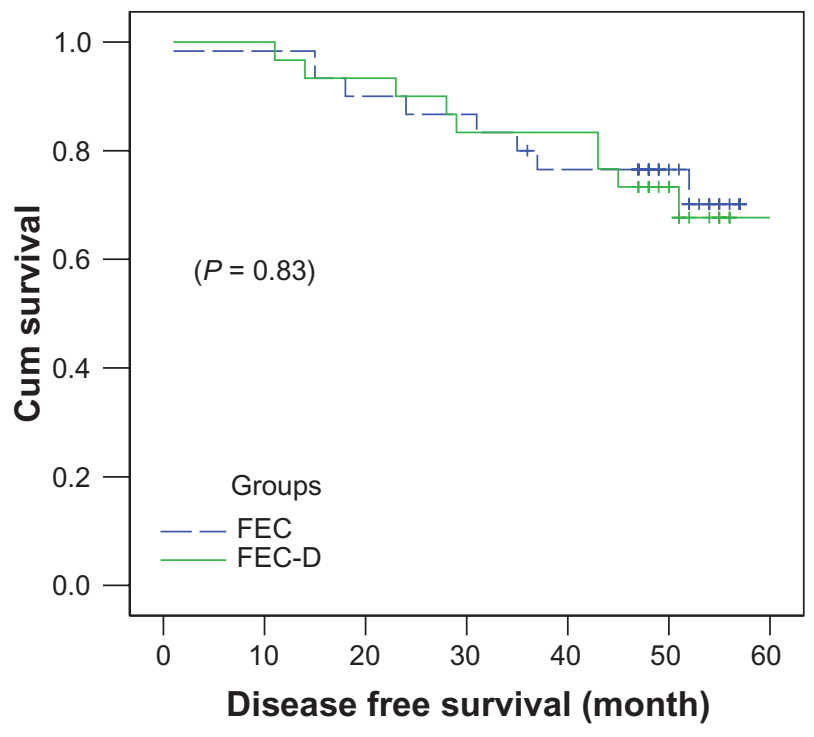

Figure I Four-year disease-free survival rates in patients receiving FEC-D or FEC-100. Abbreviations: FEC, Fluorouracil, Epirubicin, Cyclophosphamide; FEC-D, Fluorouracil, Epirubicin, Cyclophosphamide - Docetaxel.

\section{Acute and delayed toxic effects}

Grade III-IV drug-related toxicity according to National Cancer Institute Common Toxicity Criteria for Adverse Events version 3.0 was as follows: neutropenia grade III-IV was slightly higher, not reaching statistical significance in the FEC $\operatorname{arm}(5 / 30)$ versus the FEC-D arm $(4 / 30)(P=0.72)$. These patients required secondary prophylaxis by Granulocyte colony-stimulating factor (G-CSF) with subsequent cycles, as primary prophylaxis was not permitted from the beginning of treatment. Febrile neutropenia requiring hospital admission was noted in three of 30 patients in Arm I and three of

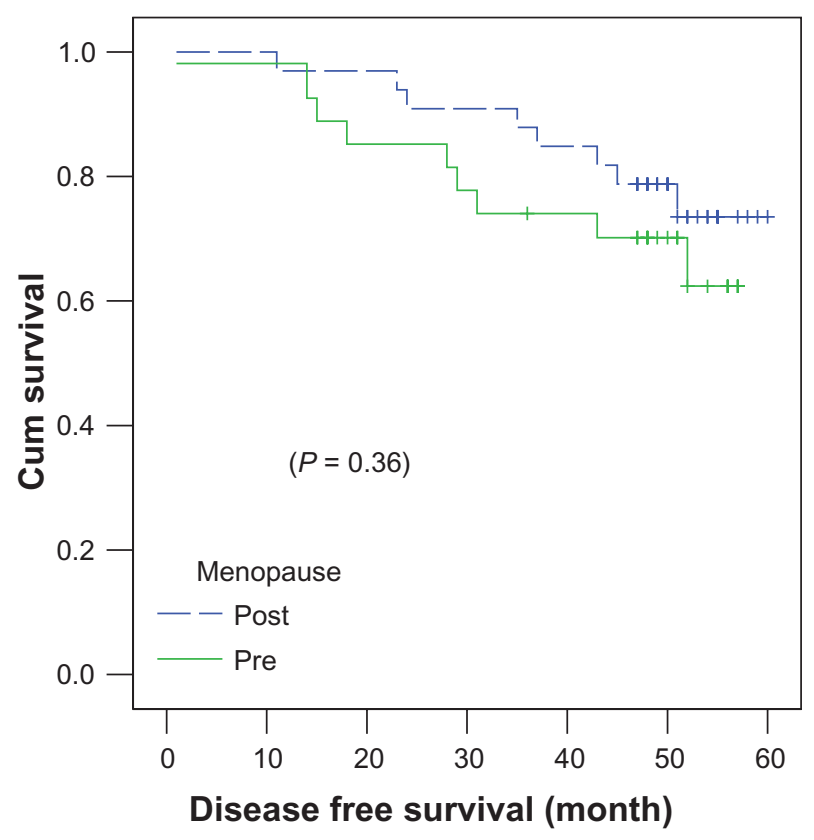

Figure 2 Menopausal status correlation with 4-year disease-free survival. 


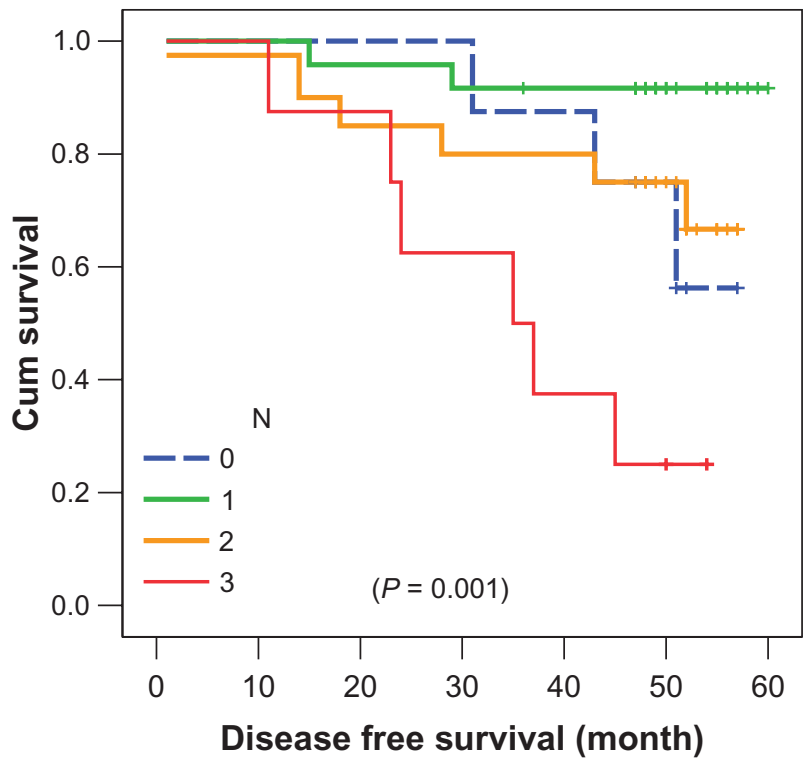

Figure 3 Nodal status correlation 4-year disease-free survival.

30 patients in Arm II $(P=1)$. Furthermore, the incidence of grade III-IV nausea and vomiting were higher in the FEC $\operatorname{arm}(6 / 30)$ versus the FEC-D arm $(4 / 30)(P=0.49)$. Hematological malignancy was not monitored in either arm, and cardiac events were not observed during follow-up; however, a decrease of $>20 \%$ of baseline value in LVEF at the end of chemotherapy occurred in two of 30 patients in Arm II versus 0 patients in Arm I $(P=0.49)$. During follow-up after the end of treatment, an echocardiography was not performed.

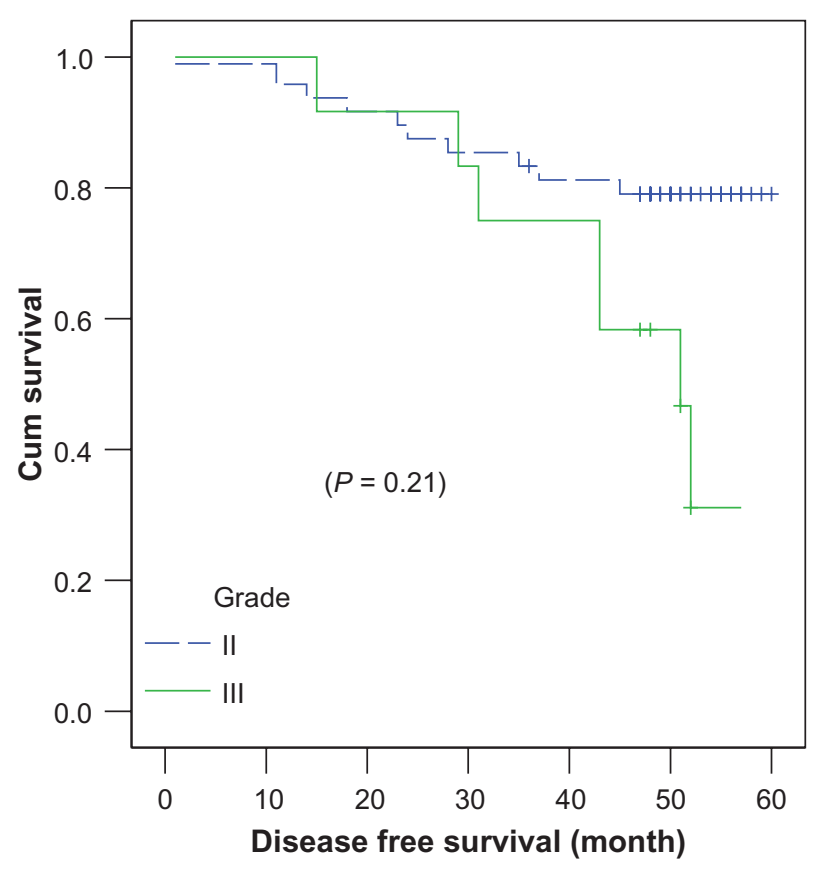

Figure 4 Tumor grade correlation with 4-year disease-free survival.

\section{Discussion}

The benefits of adjuvant taxane need to be further explored in breast cancer phenotypes, especially in the luminal A subtype. The Cancer and Leukemia Group B (CALBG) 9344 trial demonstrated benefits from paclitaxel, especially in ER-negative or HER2-positive tumors, with less benefit in luminal A. ${ }^{10}$ Other studies demonstrated improved outcomes from adjuvant sequential paclitaxel in all tumor phenotypes. ${ }^{12,13}$ The Taxotere as Adjuvant Chemotherapy Trial (TACT) included 4,162 patients and revealed that a docetaxel-based regimen was not superior to anthracycline chemotherapy of equivalent duration in terms of DFS in all tumor phenotypes. ${ }^{5}$ Another trial demonstrated benefits of adjuvant docetaxel in the whole breast cancer population. ${ }^{14}$ Of interest is the Program Action Concertée Sein-01 (PACS-01) trial, ${ }^{6}$ which included 1,999 node-positive breast cancer patients and compared six cycles of FEC-100 with three cycles of FEC-100 followed by three cycles of docetaxel $100 \mathrm{mg} / \mathrm{m}^{2}$. The result of the PACS-01 trial showed a small but significant improvement in DFS and OS. In a preplanned subgroup analysis, no benefit was seen in patients aged less than 50 years. ${ }^{6}$ Bria et al performed a pooled analysis of 15,500 patients treated in a randomized trial designed to determine if the addition of paclitaxel or docetaxel to conventional anthracycline treatment improved survival. ${ }^{15}$ The authors concluded that taxane-based sequential treatment added a significant benefit to both DFS and OS. However, the magnitude of the benefit was not large, with an approximately $2 \%-4 \%$ absolute benefit, meaning that 35 to 45 patients need to be treated for one to benefit. ${ }^{15}$ Hence, our study was designed to explore the role of the taxane docetaxel in luminal A disease (pT1-2 pN0-3 M0).

Ultimately, the present study did not show a 4-year DFS benefit from adjuvant docetaxel, coinciding with conclusions from the TACT study ${ }^{5}$ and CALBG- $9344^{10}$ in a luminal A subgroup of patients. The 4-year DFS between both groups in our study were similar, ranging from $73.3 \% \pm 8.1 \%$ for Arm I to $76.5 \% \pm 7.8 \%$ for $\operatorname{Arm}$ II $(P=0.83)$. This DFS rate is similar to that reported in the PACS-01 trial, ${ }^{6}$ which demonstrated a 5-year DFS ranging from $73.2 \%$ with FEC and $78.4 \%$ with FEC-D. The PACS-01 study reported a benefit from taxanes that reached statistical significance compared to classic FEC-100 in the 5-year DFS; however, all tumor phenotypes were included. On the other hand, the TACT trial, ${ }^{5}$ including all tumor phenotypes, reported 5-year DFS ranging from $74.3 \%$ to $75.6 \%$, with no observed benefit in 5-year DFS from adjuvant taxane $(P=0.62)$, coinciding with the results of the present study.

In terms of toxicity profile, grade III-IV nausea and vomiting were numerically higher in the FEC-100 arm, not 
reaching statistical significance $(P=0.49)$, which coincides with the toxicity incidence data reported in the PACS-01 trial, wherein a higher rate of grade III-IV nausea and vomiting in the continuous FEC arm versus the sequential docetaxel arm was found $(P=0.001)$, being more statistically significant due to their larger sample size. ${ }^{6}$ To the contrary, Gastrointestinal tract (GIT) toxicity, like nausea and vomiting, toxicity in the TACT trial was higher in the FEC-D sequential arm $(P=0.001) .{ }^{5}$ Grade III-IV hematological toxicity was higher in our study in the FEC-100 arm, but did not reach statistical significance $(P=0.72)$, coinciding with the data from TACT not reaching statistical significance, ${ }^{5}$ though grade III-IV hematological toxicity was statistically higher $(P=0.008)$ in the FEC-100 arm in the PACS trial, ${ }^{6}$ possibly due to their larger sample size.

\section{Conclusion}

This study did not support the idea that "one size fits all" in terms of DFS benefit for adjuvant docetaxel in breast cancer. Our study, focusing on the luminal A subtype, demonstrated similar DFS between FEC-100 and FEC-D treatment, where the value of taxane became less than that witnessed in other adjuvant trials recruiting all patient phenotypes. The toxicity profile was comparable between both treatment arms but numerically favored the FEC-D arm.

\section{Disclosure}

The authors report no conflicts of interest in this work.

\section{References}

1. [No authors listed]. Polychemotherapy for early breast cancer: an overview of the randomised trials. Early Breast Cancer Trialists' Collaborative Group. Lancet. 1998;352:930-942.

2. Early Breast Cancer Trialists' Collaborative Group (EBCTCG). Effects of chemotherapy and hormonal therapy for early breast cancer on recurrence and 15-year survival: an overview of the randomised trials. Lancet. 2005;365:1687-1717.
3. Ferguson T, Wilcken N, Vagg R, Ghersi D, Nowak AK. Taxanes for adjuvant treatment of early breast cancer. Cochrane Database Syst Rev. 2007;(4):CD004421.

4. De Laurentiis M, Cancello G, D’Agostino D, et al. Taxane-based combinations as adjuvant chemotherapy of early breast cancer: a meta-analysis of randomized trials. J Clin Oncol. 2008;26:44-53.

5. Hopwood P, Ridolfi A, Russell S, et al. Impact on quality of life (QoL) of FEC-T compared with FEC or CMF: UK Taxotere as Adjuvant Chemotherapy Trial (TACT) 2-year follow-up. J Clin Oncol. 2008;26(Suppl: abstr 548).

6. Roché H, Fumoleau P, Spielmann M, et al. Sequential adjuvant epirubicin-based and docetaxel chemotherapy for node-positive breast cancer patients: the FNCLCC PACS 01 Trial. J Clin Oncol. 2006;24: 5664-5671.

7. Martin M, Mackey J, Vogel C. Benefit from adjuvant taxanes and endocrine responsiveness in breast cancer. Breast. 2007;16 Suppl 2: S127-S131.

8. Andre F, Broglio K, Roche H, et al. Estrogen receptor expression and efficacy of docetaxel containing adjuvant chemotherapy in patients with node positive breast cancer: results from a pooled analysis. J Clin Oncol. 2008;26:2636-2643.

9. Hugh J, Hanson J, Cheang MC, et al. Breast cancer subtypes and response to docetaxel in node-positive breast cancer use of an immunohistochemical definition in the BCIRG 001 Trial. JClin Oncol. 2009;27: $1168-1176$.

10. Hayes DF, Thor AD, Dressler LG, et al; Cancer and Leukemia Group B (CALGB) Investigators. HER2 and response to paclitaxel in nodepositive breast cancer. N Engl J Med. 2007;357:1496-1506.

11. Cancer Therapy Evaluation Program. Common terminology criteria for adverse events v 3.0 (CTCAE). 2006. Available from: http://www. eortc.be/services/doc/ctc/ctcaev3.pdf. Accessed February 28, 2013.

12. Henderson IC, Berry DA, Demetri GD, et al. Improved outcomes from adding sequential paclitaxel but not from escalating doxorubicin dose in an adjuvant chemotherapy regimen for patients with node-positive primary breast cancer. J Clin Oncol. 2003;21:976-983.

13. Mamounas EP, Bryant J, Lembersky B, et al. Paclitaxel after doxorubicin plus cyclophosphamide as adjuvant chemotherapy for node-positive breast cancer: results from NSABP B-28. J Clin Oncol. 2005;23:3686-3696.

14. Martin M, Pienkowski T, Mackey J, et al; Breast Cancer International Research Group 001 Investigators. Adjuvant docetaxel for node-positive breast cancer. $N$ Engl J Med. 2005;352:2302-2313.

15. Bria E, Nistico C, Cuppone F, et al. Benefits of taxane as adjuvant chemotherapy for early breast cancer: pooled analysis of 15,500 patients. Cancer. 2006;106(11):2337-2344.
OncoTargets and Therapy

\section{Publish your work in this journal}

OncoTargets and Therapy is an international, peer-reviewed, open access journal focusing on the pathological basis of all cancers, potential targets for therapy and treatment protocols employed to improve the management of cancer patients. The journal also focuses on the impact of management programs and new therapeutic agents and protocols on

\section{Dovepress}

patient perspectives such as quality of life, adherence and satisfaction. The manuscript management system is completely online and includes a very quick and fair peer-review system, which is all easy to use. Visit http://www.dovepress.com/testimonials.php to read real quotes from published authors. 